\title{
Entropy Production Estimates For a Total Variation Image Model
}

\author{
Lihua Min ${ }^{1, a^{*}}$, Can Feng ${ }^{2, b}$ and Zhengmeng $\operatorname{Jin}^{1, c}$ \\ ${ }^{1}$ School of Science, Nanjing University of Posts and Telecommunications, Nanjing, 210023, China \\ ${ }^{2}$ North Information Control Academe Group Co. Ltd., Nanjing, 211153, China \\ amlh@njupt.edu.cn, bengcan2004@126.com, Cjinzhm@njupt.edu.cn
}

Keywords: Image processing; Entropy production estimates; Radial solution; Total Variation

\begin{abstract}
The main aim of this paper is to give some theoretical analysis for a nonlinear higher order total variation image model. Applying the algebraic approach, we transform the analytical problem of proving entropy dissipation inequality into an algebraic problem about the nonnegativity of certain polynomial. As a result, we derive some key entropy production estimates of radial symmetric solutions in higher dimensions, which improve and extend some previous results.
\end{abstract}

\section{Introduction}

In the last decades, nonlinear partial differential equations (PDEs) are commonly used in image processing. In this paper, we continue to investigate the following nonlinear fourth order PDE

$$
u_{t}+\Delta\left(u^{-1} \Delta u\right)=0
$$

Eq.1 is an effective model for noise removal in image processing ${ }^{[1]}$ and corresponds to the famous total variation diffusivity ${ }^{[2,3,4, \text { and } 5]}$. The one dimensional case of the initial boundary value problem of Eq. 1 was discussed in ${ }^{[1,6]}$, based on the entropy dissipation method. The term "entropy" is widely defined in various subjects and frequently used for a positive Lyapunov functional in mathematics ${ }^{[6-10]}$ : Suppose that a PDE for the variable $U=U(x, t)$ possesses positive functional $E(U)$, such that

$$
E(U(x, t))+\delta_{0} \int_{0}^{t} P\left(U(x, \tau), U_{x}(x, \tau), \cdots\right) d \tau \leq E(U(x, 0)),
$$

For a constant $\delta_{0}>0$, then functional $E(U(x, t))$ is called an entropy with $P>0$ which is the corresponding entropy production. The inequality (2.1) is called entropy dissipation estimates, which can be used to estimate the large time behavior of solutions.

For a model with practical significance, it is natural to investigate it in higher dimensions. Recently, the author ${ }^{[11]}$ proved that there also exist entropies for Eq.1 in higher dimensions. Let $U(x, t)=u(r, t)$ with $r=|x|$ be a smooth radially symmetric solution of the following fourth order nonlinear degenerate problem

$$
\left\{\begin{array}{l}
U_{t}+\Delta\left(U^{-1} \Delta U\right)=0, \quad x \in \Omega=B^{N}, t>0, \\
\nabla U \cdot v=\nabla\left(U^{-n} \Delta U\right) \cdot v=0, \quad x \in \partial \Omega, t>0, \\
U(x, 0)=U_{0}(|x|) \quad x \in \Omega,
\end{array}\right.
$$

where $B^{N}$ is a unit ball in $R^{N}(N \geq 1), \quad v$ is the outer normal vector at the boundary of the unit sphere, and let 


$$
E_{\alpha}(U(x, t))=\int_{0}^{1} \omega_{N} e(u(r, t)) r^{N-1} d r
$$

Then ${ }^{[6,11]}$ the functional $E(t):=E_{\alpha}(U(x, t))$, defined in (4) is an entropy which is nonincreasing in time $\mathrm{t}$ if $1 \leq \alpha \leq 4$ for $N=1$ and $\frac{3 N+4-4 \sqrt{N}}{N+2} \leq \alpha \leq 4$ for $N=2,3$. Furthermore, there holds

$$
u_{t}(r, t)=-\nabla_{x} \cdot\left\{\nabla_{x}\left[u^{-n}\left(u_{r r}+\frac{N-1}{r} u_{r}\right)\right]\right\} \square-\nabla_{x} \cdot\left\{F(r) \cdot \frac{x}{r}\right\},
$$

where

$$
F(r)=\left[u^{-n}\left(u_{r r}+\frac{N-1}{r} u_{r}\right)\right]_{r}=u^{-n+1}\left\{\frac{u_{r r r}}{u}-n \frac{u_{r}}{u} \frac{u_{r r}}{u}+\frac{N-1}{r}\left[\frac{u_{r r}}{u}-n\left(\frac{u_{r}}{u}\right)^{2}-\frac{u_{r}}{r u}\right]\right\}
$$

here we have identified $\left(\frac{\partial_{r}^{i} u}{u}\right)^{m}=\eta_{i}^{m}(i=1,2,3)$ and $\xi=\frac{1}{r}$ for simplification.

This paper continues to consider the entropy production estimates to the radial solution of problem (3). The main results are as follows:

Theorem. Let $U(x, t)=u(r, t)$ be a positive radially symmetric smooth solution of problem (4), $N=2,3$, then the entropy dissipation inequality (2) holds with

$$
P(U)=\int_{\Omega}\left(\Delta U^{\frac{\alpha-1}{2}}\right)^{2} d x \quad(\alpha \neq 1)
$$

and

$$
0<\delta_{0} \leq \begin{cases}\frac{4\left[(\alpha-3)^{2} N^{2}+4(\alpha-2)^{2}+4 N\left(\alpha^{2}-5 \alpha+2\right)\right]}{(\alpha-1)^{3}(N+2)(\alpha N-5 N+2 \alpha-6)}, & \text { if } \frac{3 N+4-4 \sqrt{N}}{N+2}<\alpha \leq \frac{13 N+20}{3(N+2)}, \\ \frac{32(4-\alpha)}{(\alpha-1)^{3}}, & \text { if } \frac{13 N+20}{3(N+2)}<\alpha<4 .\end{cases}
$$

The following two lemmas will be used in the proof of main results.

\section{Lemma 1[12] Let}

$$
P\left(\xi, \eta_{1}, \eta_{2}\right)=a_{1} \eta_{1}^{4}+a_{2} \eta_{1}^{2} \eta_{2}+a_{3} \eta_{2}^{2}+a_{4} \xi \eta_{1}^{3}+a_{5} \xi^{2} \eta_{1}^{2}+a_{6} \xi \eta_{1} \eta_{2},
$$

Be a polynomial with real coefficients. Then the quantified formula

$$
\forall\left(\xi, \eta_{1}, \eta_{2}\right) \in R^{3}: P\left(\xi, \eta_{1}, \eta_{2}\right) \geq 0
$$

is equivalent to either $a_{3}=0$ and $a_{2}=a_{6}=0$ and $\left[\left(4 a_{1} a_{5}-a_{4}^{2} \geq 0, a_{5}>0\right)\right.$ or $\left.\left(a_{1} \geq 0, a_{4}=a_{5}=0\right)\right]$; 
or $a_{3}>0$ and $\left[\left(4 a_{3} a_{5}-a_{6}^{2}>0,4 a_{1} a_{3} a_{5}-a_{3} a_{4}^{2}-a_{2}^{2} a_{5}-a_{1} a_{6}^{2}+a_{2} a_{4} a_{6} \geq 0\right)\right.$ or $\left(4 a_{1} a_{3}-a_{2}^{2} \geq 0\right.$,
$\left.\left.2 a_{3} a_{4}-a_{2} a_{6}=4 a_{3} a_{5}-a_{6}^{2}=0\right)\right]$.

Lemma $2^{[12]}$ let the polynomial $P(x)=\underline{b}_{2}^{2}+b \not{ }_{c}{ }_{\text {with }} b_{2} \geq 0$, and real numbers $z_{1}<z_{2}$ be given. Then the quantified formula is equivalent to either

$$
\begin{aligned}
& b_{2}=0 \text { and }\left[\left(b_{1}<0, b_{1} z_{2}+b_{0}<0\right) \text { or }\left(b_{1}>0, b_{1} z_{1}+b_{0}<0\right) \text { or }\left(b_{1}=0, b_{0} \leq 0\right)\right] ; \\
& \text { or } b_{2}>0 \text { and }\left[b_{2} z_{1}^{2}+b_{1} z_{1}+b_{0}<0 \text { or }\left(b_{1}+2 b_{2} z_{1}<0, b_{1}^{2}-4 b_{0} b_{2} \geq 0\right)\right] \\
& \text { and }\left[b_{2} z_{2}^{2}+b_{1} z_{2}+b_{0}<0 \text { or }\left(b_{1}+2 b_{2} z_{2}>0, b_{1}^{2}-4 b_{0} b_{2} \geq 0\right)\right] .
\end{aligned}
$$

\section{Sketch Proof of Main results}

In this section, following the ideals in [6, 12], we prove our main theorem. Firstly [11] for any $c_{1}, c_{2} \in R$,

$$
\frac{d}{d t} E_{\alpha}(U(x, t))=-\omega_{N} \int_{0}^{1} u^{\alpha-1} S_{c_{1}, c_{2}}(\xi, \eta) r^{N-1} d r
$$

here $S_{c_{1}, c_{2}}(\xi, \eta)=b_{1} \eta_{1}^{4}+b_{2} \eta_{1}^{2} \eta_{2}+b_{3} \eta_{2}^{2}+b_{4} \xi \eta_{1}^{3}+b_{5} \xi^{2} \eta_{1}^{2}+b_{6} \xi \eta_{1} \eta_{2}$, for $b_{1}=c_{1}(\alpha-4)$,

$$
b_{2}=3 c_{1}+\alpha-2, b_{3}=1, \quad b_{4}=c_{2}(\alpha-3)+(N-1)\left(c_{1}+1\right), b_{5}=c_{2}(N-2)+(N-1), \quad b_{6}=2 c_{2} .
$$

On the other hand, entropy production

$$
P(U)=\int_{\Omega}\left(\Delta U^{\frac{\alpha-1}{2}}\right)^{2} d x=\omega_{N} \int_{0}^{1} u^{\alpha-1} S_{p}(\xi, \eta) r^{N-1} d r
$$

where

$$
\begin{aligned}
& S_{p}(\xi, \eta)=\left(\frac{\alpha-3}{2}\right)^{2}\left(\frac{\alpha-1}{2}\right)^{2} \eta_{1}^{4}+(\alpha-3)\left(\frac{\alpha-1}{2}\right)^{2} \eta_{1}^{2} \eta_{2}+\left(\frac{\alpha-1}{2}\right)^{2} \eta_{2}^{2}+(\alpha-3)\left(\frac{\alpha-1}{2}\right)^{2}(N-1) \xi \eta_{1}^{3} \\
& +\left(\frac{\alpha-1}{2}\right)^{2}(N-1)^{2} \xi^{2} \eta_{1}^{2}+2\left(\frac{\alpha-1}{2}\right)^{2}(N-1) \xi \eta_{1} \eta_{2} .
\end{aligned}
$$

We now quantify the positive constant $\delta_{0}$ for which there exist $c_{1}, c_{2}$ and for any $\left(\xi, \eta_{1}, \eta_{2}\right) \in R^{3}$, making

$$
S_{\delta_{0}}(\xi, \eta)=d_{1} \eta_{1}^{4}+d_{2} \eta_{1}^{2} \eta_{2}+d_{3} \eta_{2}^{2}+d_{4} \xi \eta_{1}^{3}+d_{5} \xi^{2} \eta_{1}^{2}+d_{6} \xi \eta_{1} \eta_{2} \geq 0
$$

here 


$$
\begin{aligned}
& d_{1}=c_{1}(\alpha-4)-\delta_{0}\left(\frac{\alpha-3}{2}\right)^{2}\left(\frac{\alpha-1}{2}\right)^{2}, \quad d_{2}=3 c_{1}+\alpha-2-\delta_{0}(\alpha-3)\left(\frac{\alpha-1}{2}\right)^{2}, \quad d_{3}=1-\delta_{0}\left(\frac{\alpha-1}{2}\right)^{2}, \\
& d_{4}=(N-1)\left(c_{1}+1\right)+\left[c_{2}-\delta_{0}\left(\frac{\alpha-1}{2}\right)^{2}(N-1)\right](\alpha-3), \quad d_{5}=c_{2}(N-2)+(N-1)-\delta_{0}\left(\frac{\alpha-1}{2}\right)^{2}(N-1)^{2}, \\
& d_{6}=2 c_{2}-2 \delta_{0}\left(\frac{\alpha-1}{2}\right)^{2}(N-1) .
\end{aligned}
$$

According to Lemma 1, we just consider the case for $d_{3}>0 \cdot$ Hence, (11) is equivalent to either $4 d_{1} d_{3}-d_{2}^{2}=-9\left[c_{1}-\frac{\delta_{0}(\alpha-1)^{3}-8-4 \alpha}{36}\right]^{2}+\frac{1}{144}(\alpha-1)\left[\delta_{0}(\alpha-1)^{2}-4\right]\left[\delta_{0}(\alpha-1)^{3}+32(\alpha-4)\right] \geq 0$

and

$$
2 d_{3} d_{4}-d_{2} d_{6}=4 d_{3} d_{5}-d_{6}^{2}=0
$$

or

$$
0<4 d_{3} d_{5}-d_{6}^{2}=-\left(c_{2}+1-N\right)\left[4 c_{2}+4-(\alpha-1)^{2} N \delta_{0}\right] \square-M_{1},
$$

and $\quad 0 \leq 4 d_{1} d_{3} d_{5}-d_{3} d_{4}^{2}-d_{2}^{2} d_{5}-d_{1} d_{6}^{2}+d_{2} d_{4} d_{6}$

$$
\begin{aligned}
& =\frac{c_{1}^{2}}{4-\delta_{0}(\alpha-1)^{2}}\left\{9 M_{1}-\left[\left(\delta_{0}(\alpha-1)^{2}+2\right)(N-1)-6 c_{2}\right]^{2}\right\}+\left[2\left(c_{2}+1-N\right)(N+2)+\frac{M_{1}}{2}(\alpha-1)\right] c_{1} \\
& +N\left(c_{2}+1-N\right)+\frac{M_{1}}{4}\left(\alpha^{2}-4 \alpha+3\right) .
\end{aligned}
$$

Lemma 2 is used and gives that

$$
0<\delta_{0} \leq \begin{cases}\frac{4\left[(\alpha-3)^{2} N^{2}+4(\alpha-2)^{2}+4 N\left(\alpha^{2}-5 \alpha+2\right)\right]}{(\alpha-1)^{3}(N+2)(\alpha N-5 N+2 \alpha-6)}, & \text { if } \frac{3 N+4-4 \sqrt{N}}{N+2}<\alpha \leq \frac{13 N+20}{3(N+2)}, \\ \frac{32(4-\alpha)}{(\alpha-1)^{3}}, & \text { if } \frac{13 N+20}{3(N+2)}<\alpha<4 .\end{cases}
$$

We conclude the proof.

\section{Conclusions}

This paper gave some theoretical analysis for a nonlinear higher order total variation image model. Applying the algebraic approach, we transform the analytical problem of proving entropy dissipation inequality into an algebraic problem about the nonnegativity of certain polynomial. The results obtained improve and extend some previous conclusions.

\section{Acknowledgements}

This paper is partially supported by the National Natural Science Foundation of China (No. 11401318, No. 11671004), the Natural Science Foundation of the Jiangsu Higher Education Institutions of China (No.14KJB110020, No.15KJB110018) and the Scientific Research Foundation of NUPT (No.NY214023). 


\section{References}

[1] L. H. Min, X. P. Yang, D. Ye, Well-posedness for a fourth order nonlinear equation related to image processing, Nonlinear Anal. Real World Appl. 17 (2014) 192-202.

[2] S. Didas, J. Weickert, B. Burgeth, Properties of higher order nonlinear diffusion filtering, J. Math. Imaging Vision. 35(3) (2009) 208-226.

[3] Y. L. Wang, J. F. Yang, W. T. Ying, Y. Zhang, A new alternating minimization algorithm for total variation image reconstruction, SIAM J. Imaging Sci. 1 (2) (2008) 248-272.

[4] T. Chan, A. Marquina, P. Mulet, High-order total variation-based image restoration, SIAM J Sci Compute. 22(2000) 503-516.

[5] S. Didas, J, Weickert, B. Burgeth, Properties of higher order nonlinear diffusion filtering [J]. J Math Imaging Vis. 35(2009)208-226.

[6] L. H. Min, X. P. Yang, C. F. Gui, Entropy estimates and large time behavior of solutions to a fourth order nonlinear degenerate equation, Commun. Contemp. Math., 15(4) (2013) 1250066 (23 pages).

[7] L. H. Min, X. P. Yang, Finite speed of propagation and algebraic time decay of solutions to a generalized thin film equation, Commun. Pure Appl. Anal. 13(2) (2014) 543-566.

[8] J. A. Carrillo, A. Jungel, P. A. Markowich, G. Toscani, A. Unterreiter, Entropy dissipation methods for degenerate parabolic problems and generalized Sobolev inequalities, Monatsh. Math. 133(2001) 1-82.

[9] A. Jungel, G. Toscani, Exponential time decay of solutions to a nonlinear fourth-order parabolic equation, Z. Angew. Math. Phys. 54(2003) 377-386.

[10] F. Bernis, A. Friedman, Higher-order nonlinear degenerate parabolic equations, J. Differential Equations. 83 (1990) 179-206.

[11] L. H. Min, Entropy estimates of radial symmetric solutions to a fourth order nonlinear degenerate equation in higher dimensions, Int. J. Contemp. Math. Sciences. 10(5) (2015) 209-221.

[12] M. Bukal, A. J“ungel, D. Matthes, Entropies for radially symmetric higher-order nonlinear diffusion equations, Commun. Math. Sci. (18)9(2010) 353-382. 